detection of p24 antigen with the potential to enhance diagnostic sensitivity in early infections. Future research should focus on better combination antigen/antibody rapid assays or rapid tests to detect nucleic acid to improve the diagnosis of early HIV seroconversion and for early infant diagnosis. Another area of unmet need is the accurate detection of infection in individuals who have received HIV vaccine who may screen positive on rapid tests making distinguishing vaccination and disease difficult. Finally, future directions should combine testing for multiple infections (e.g. HIV, HBV, HCV and syphilis) in a single test kit.

\section{S02.3 EXTRA-GENITAL CT/GC TESTING BY NAATS: USE IN SCREENING AND PREVALENCE OF INFECTION IN MSM, ISSUES IN VALIDATION}

doi:10.1136/sextrans-2013-051184.0018

J Schachter. UCSF, San Francisco, CA, United States

Most Chlamydia trachomatis (CT) and Neisseria gonorrhoeae (GC) infections in men who have sex with men (MSM) are not in the urethra. This has been confirmed often since it was shown by Kent and colleagues, in 2 clinics in San Francisco, (J. Infect. Dis. 2003). All of these studies have been made possible by the use of highly sensitive and specific nucleic acid amplification tests (NAATs) that are currently recommended for routine diagnosis of CT/GC infections. The increment in sensitivity for NAATs compared to culture is greater with pharyngeal and rectal specimens than with cervical and urethral specimens; doubling the number of rectal or pharyngeal infections detected.

In MSM attending STD clinics the prevalence of rectal or urethral CT and GC is often in the $7-10 \%$ range. GC is found in the oropharynx at about the same level, but CT is less common there, typically $1-2 \%$. Most STD clinics' routine has been to test urethral specimens when evaluating males, with rectal or oropharyngeal specimens tested in symptomatic MSM. We need a paradigm shift: in MSM routine testing of oropharyngeal and rectal sites, as well as urethra, must become the norm. Whether testing should be based on a history of sex practises needs more research.

Unfortunately, no NAATs have received FDA clearance for pharyngeal or rectal specimens. But CDC, recognising the superior performance of NAATs with these specimens, took an unusual step recommending NAATs for diagnosing CT/GC in oropharynx and rectum, despite absence of FDA clearance.

It is possible to use tests that have not received FDA clearance for patient management. Large laboratories can verify use of NAATs for rectal and pharyngeal specimens by following Clinical Laboratory Improvement Act (CLIA) guidelines. We need NAATs with FDA clearance for use on oropharyngeal and rectal specimens to further expand clinical access to these tests.

\section{SO2.4 WHAT ARE THE MOST EFFICIENT WAYS TO COMMUNICATE RESEARCH DERIVED INFORMATION TO CLINICAL PRACTISE: THE ROLE OF DIAGNOSTIC AND TREATMENT GUIDELINES, PACKET INSERTS, CLINICIANS' PERSPECTIVE}

doi:10.1136/sextrans-2013-051184.0019

\section{J White. Guy's and St Thomas' Hospitals, London, UK}

When implementing innovative diagnostics or novel therapeutics in medicine, the modern process of knowledge transfer can be described in a stepwise progression from (1) knowledge creation, to (2) diffusion and dissemination, and finally (3) organisational adoption and implementation. We can observe how such processes have contributed to the STI testing and treatment guidelines that we use currently.

As clinicians we strive to keep abreast of developments in our field that enable us to deliver the best quality management to our patients. The experience and intuition of an individual clinician is valuable, but all our practises should be subject to rigorous evaluation to ensure they are safe and effective. Without such processes, different and conflicting practises can persist, examples of which will be discussed. The need to implement evidence-based best practise has become widely accepted; however, this can still be hampered by financial constraints as well as the unavailability of specific resources locally. In addition, there is often a lag time in our ability to adopt new tests or treatments due to the need to adhere to local or national guidelines or a lack of evidence such as randomised controlled trials that drive changes in practise.

This symposium allows us to examine the most effective ways of getting research into practise and this session will focus on the clinician's viewpoint. Many knowledge gaps remain where further work is needed to better guide STI testing and treatment. More consideration is needed as to how new information is best disseminated to enable our patients to benefit most promptly from implementation of new information.

\section{S.03 - Advancing prevention of sexually transmitted infections through sexual health promotion: Opportunities and lessons learned (organised by CDC/WHO/ECDC)}

\section{S03.1 SEXUAL HEALTH: CONCEPTUAL FRAMEWORK AND RECOMMENDATIONS FOR INDICATORS}

doi:10.1136/sextrans-2013-051184.0020

II A Toskin, ${ }^{2} \mathrm{~S}$ Hawkes, ${ }^{1} \mathrm{C}$ Garcia Moreno, ${ }^{3} \mathrm{C}$ F Caceres, ${ }^{4} \mathrm{~L}$ Zohrabyan. ${ }^{\top}$ World Health Organization, Geneva, Switzerland; Institute of Global Health, University College London, London, UK; ${ }^{3}$ Universidad Peruana Cayetano Heredia, Instituto de Estudios en Salud, Sexualidad y Desarrollo Humano, Lima, Peru; ${ }^{4}$ UNAIDS RST ECA, Moscow, Russian Federation

Background In 2010 WHO convened an expert consultation to formulate recommendations and strategic directions for sexual health. Two specific recommendations, derived from the consultation were: (a) to develop a conceptual framework on sexual health that clearly outlines the elements of sexual health and how it overlaps and differs from reproductive health and the role of sexuality; (b) to develop, operationalize and promote sexual health indicators. Method The WHO Department of Reproductive Health and Research established consultative processes, including a review of the existing evidence, conducted interviews with key informants and held expert consultations to address the aforementioned recommendations.

Results Two documents; Towards a conceptual framework for sexual health: understanding and improving sexual health for all and Core Set of Sexual Health Indicators were developed during 2011-2013.

The conceptual framework outlines the central role that key sexual health concepts of autonomy, individual choice and protection of human rights play in achieving health and development outcomes. The document proposes new ways of 'framing' sexual health in order to reach the widest audience, which in turn can influence and deliver positive approaches for ensuring sexual health for all.

The proposed indicators cover the following areas of sexual health: adolescent sexual health, family planning, harmful practises, healthy sexuality, sexual dysfunctions and concerns, STI/HIV, and sexual violence. Indicators range from policy, to services (access) to outcome/impact. Most of the proposed indicators have previously been validated, however some new population-based survey indicators have been submitted for validation through special surveys among men who have sex with men and people who inject drugs, to be conducted throughout 2012/2013 in the WHO European region. Preliminary validation results are available. 